\title{
The Effect Of Transport Protocol Performance On Encrypted Traffic Through Ad Hoc Networks
}

\author{
Andi Gunanta Ginting \\ Department Of Electrical Engineering, University Of North Sumatra, Jl. Dr. Mansur No. 9 Padang Bulan, Kec. \\ Medan Baru, Kota Medan 20222
}

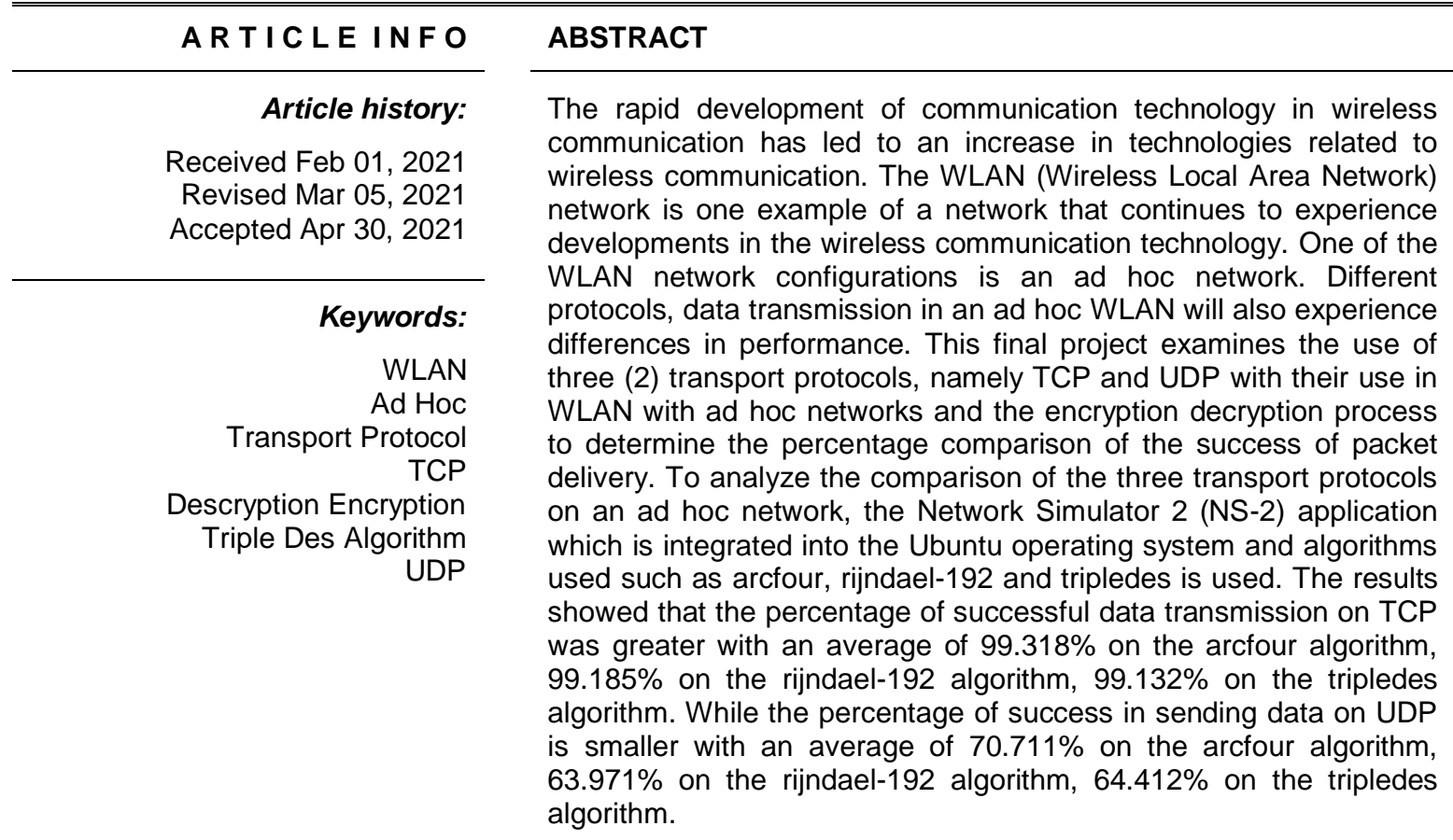

This is an open access article under the CC BY-NC license.

\section{Corresponding Author:}

Andi Gunanta Ginting ,

Department Of Electrical Engineering,

University Of North Sumatra,

JI. Dr. Mansur No. 9 Padang Bulan, Kec. Medan Baru, Kota Medan 20222.

Email : andiginting@gmail.com

\section{INTRODUCTION}

Transport layer protocols in the TCP/IP protocol suite often experience problems in wireless networks. This is due to the high packet loss on the network. On the other hand, the security in a wireless network is lower than in a wired network, because the signal that carries information propagates freely and other people's access to the signal cannot be restricted. Data security solutions that are often done are through encryption.

Both transmission control protocol (TCP) and user datagram protocol (UDP) have advantages and disadvantages for wireless data transmission. Delay and packet loss into consideration both. This thesis examines the effect of achieving transport layer protocols, both TCP and UDP, on encrypted data on an ad hoc wireless network. 


\section{RESEARCH METHOD}

This thesis uses a simulation approach using a network simulator to measure the effect of the performance of the transport layer on the delivery of encrypted data on the adhoc network.

\subsection{Network and Traffic Specifications}

The study was conducted using the NS-2 simulator with an ad hoc network topology. Each node is connected dynamically and can serve in the routing process. All nodes are evaluated based on the data received.

\subsection{Protocols Under Review}

The protocols evaluated are TCP and UDP. This is due to the limited research time and implementation capabilities of the protocol.

\subsection{Encryption Evaluation Method}

The encryption process is carried out before the simulation is run. Encryption is done using tools that are available open source. In this case, this thesis utilizes an online encryption algorithm on the website:https://codebeautify.org/encrypt-decrypt.

The results of the encryption are then broken into packets measuring 20 bytes, and the delivery is simulated using a network simulator to determine delay, jitter and packet loss. If the encryption method produces the same cyphertext length, then the network simulation will produce the same pattern because the simulator is deterministic, i.e. repeated simulations give the same results.

The difference is that the description of different encryption methods can produce different data. The results of the delivery, namely the data received are described, including the reduction of lost packets, using the same tools on the website: https://codebeautify.org/encrypt-decrypt. With this process, it will be known how far the success rate of encrypted data description, if sent using TCP and UDP.

\section{RESULTS AND DISCUSSIONS}

\subsection{Characteristics of Sending Encrypted Data with TCP}

Encrypted data, shredded based on TCP transport protocol capabilities. The received data is aggregated and described. The following are the results of the evaluation of sending encrypted data using TCP.

\subsection{Arcfour Encryption Method}

\section{a. Delay}

Table 1 shows the average data delay experienced by each resulting packet when the packets are sent using TCP.

Table 1. Delay of Arcfour encrypted packets

\begin{tabular}{cc}
\hline Number of Nodes & delay \\
\hline 2 & 0.002022436 \\
4 & 0.008574443 \\
6 & 0.03641429 \\
8 & 0.02601235 \\
10 & 0.02088144 \\
12 & 0.05019733 \\
14 & 0.08386823 \\
16 & 0.05234833 \\
18 & 0.08726496 \\
20 & 0.09936863
\end{tabular}


Figure 1 shows the plot of the average delay which increases with the increase in the number of nodes. The results of the regression analysis resulted in the trend equation $y=0.0052 x-0.0103$.

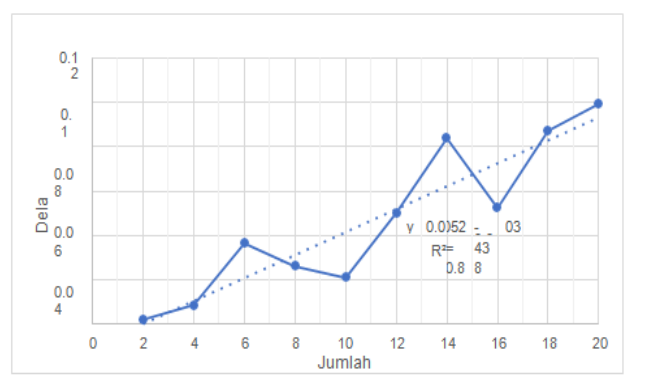

Figure 1. Average delay of Arcfour packets with TCP

\section{b. Jitter}

Table 2 shows the average data jitter experienced by each resulting packet when the packets are sent using TCP.

Table 2. Packet Jitter-package encrypted Arcfour

\begin{tabular}{cc}
\hline Number of Nodes & Jitter \\
\hline 2 & 0.00048052 \\
4 & 0.00389286 \\
6 & 0.00777959 \\
8 & 0.01154746 \\
10 & 0.01254893 \\
12 & 0.0177959 \\
14 & 0.01823111 \\
16 & 0.01811854 \\
18 & 0.04181797 \\
20 & 0.02835846 \\
\hline
\end{tabular}

Figure 2 shows the average jitter plot which increases with the increase in the number of nodes. The results of the regression analysis resulted in the trend equation $y=0.0018 x-0.0037$.

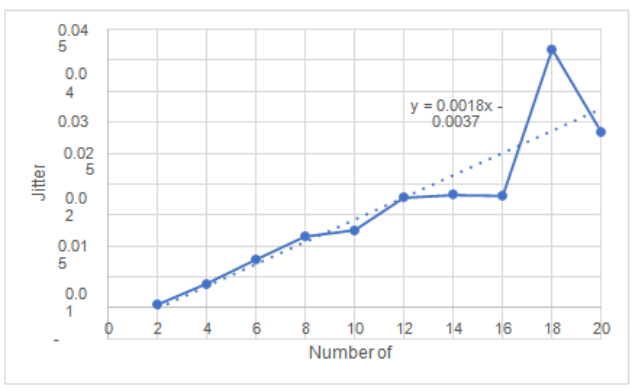

Figure 2. Average Jitter of Arcfour packets with TCP

\section{c. Packet Loss}


Table 3 shows the average packet loss data experienced by each resulting packet when the packets are sent using TCP.

Table 3. Packet Loss of Arcfour encrypted packets

\begin{tabular}{cc}
\hline Number of Nodes & Packet Loss \\
\hline 2 & $0 \%$ \\
4 & $0 \%$ \\
6 & $0 \%$ \\
8 & $0.60 \%$ \\
10 & $0.71 \%$ \\
12 & $0.86 \%$ \\
14 & $0.60 \%$ \\
16 & $0.80 \%$ \\
18 & $0.46 \%$ \\
20 & $3.30 \%$ \\
\hline
\end{tabular}

Figure 3 shows a plot of the average packet loss which increases with the increase in the number of nodes. The results of the regression analysis resulted in the trend equation $y=0.0011 x$ 0.005 .

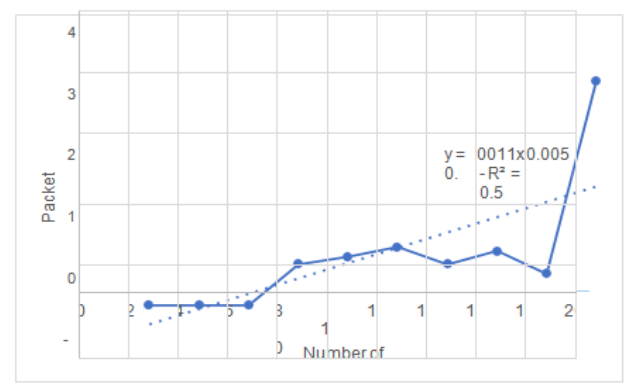

Figure 3. Average Packet Loss of Arcfour packets with TCP

\section{d. Decryption Success Rate}

The success rate of the data after being decrypted can be shown in Table 4.

Table 4. Arcfour Decryption Success Rate With TCP Protocol

\begin{tabular}{cc}
\hline Number of Nodes & Decryption Result \\
\hline 2 & $100 \%$ \\
4 & $100 \%$ \\
6 & $100 \%$ \\
8 & $99.45 \%$ \\
10 & $99.34 \%$ \\
12 & $99.34 \%$ \\
14 & $99.49 \%$ \\
16 & $99.36 \%$ \\
18 & $99.43 \%$ \\
20 & $96.77 \%$ \\
\hline
\end{tabular}

The simulation results show that, on average, the description results will decrease with the increase in the number of nodes. Figure 4 shows that the results of the regression analysis produce a trend equation $\mathrm{y}=-0.0011 \mathrm{x}+1.0052$ 


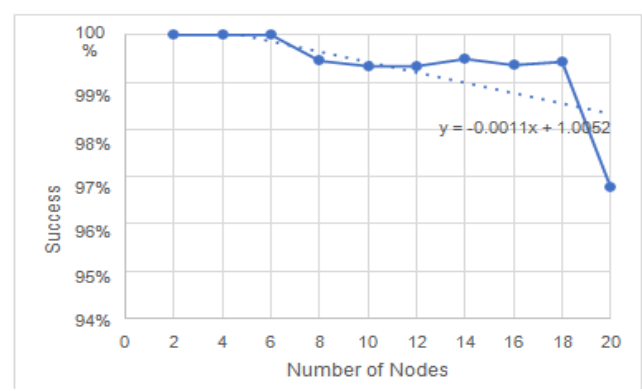

Figure 4. Arcfour Decryption Success Rate With TCP Protocol

\subsection{TripleDES Encryption Method}

\section{a. Delay}

Table 5 shows the average data delay experienced by each resulting packet when the packets are sent using UDP.

Table 5. Delay of TripleDES encrypted packets

\begin{tabular}{cc}
\hline Number of Nodes & delay \\
\hline 2 & 0.00115282 \\
4 & 0.004272687 \\
6 & 0.18288052 \\
8 & 0.0253033 \\
10 & 0.06602001 \\
12 & 0.11078979 \\
14 & 0.12708878 \\
16 & 0.0709795 \\
18 & 0.34710979 \\
20 & 0.13899728 \\
\hline
\end{tabular}

Figure 4 shows the plot of the average delay which increases with the increase in the number of nodes. The results of the regression analysis resulted in the trend equation $y=0.0162 x-0.0424$

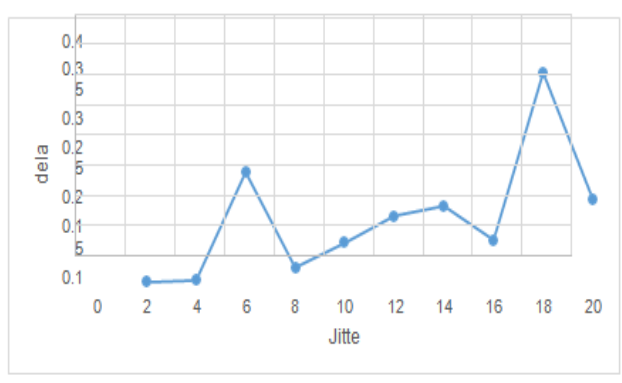

Figure 5. Average Delay of TripleDES packets with UDP

\section{b. Jitter}

Table 66 shows the average jitter data experienced by each resulting packet when the packets are sent using UDP. 
Table 6. Jitter of TripleDES encrypted packets

\begin{tabular}{cc}
\hline Number of Nodes & Jitter \\
2 & 0.00031148 \\
4 & 0.001922479 \\
6 & 0.030247414 \\
8 & 0.007150093 \\
10 & 0.009587053 \\
12 & 0.022650893 \\
\hline
\end{tabular}

Figure 5 shows the average jitter plot which increases with the increase in the number of nodes. The results of the regression analysis resulted in the trend equation $y=0.0019 x+0.0006$

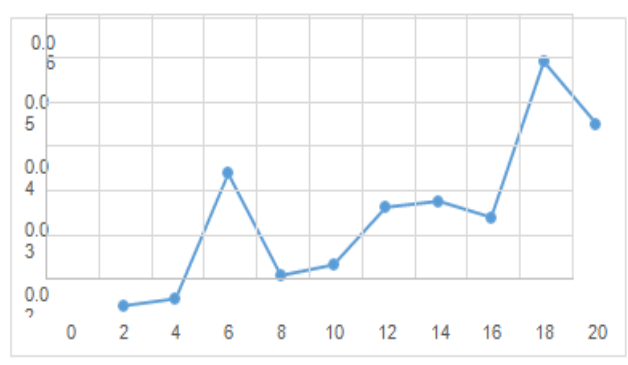

Figure 6. Average Jitter of Rijndael-192 packets with UDP

\section{c. Packet Loss}

Table 7 displays the average packet loss data experienced by each resulting packet when the packets are sent using UDP.

Table 7. Packet Loss of TripleDES encrypted packets

\begin{tabular}{cc}
\hline Number of Nodes & Packet Loss \\
\hline 2 & $1 \%$ \\
4 & $1.50 \%$ \\
6 & $1.75 \%$ \\
8 & $2.20 \%$ \\
10 & $3.67 \%$ \\
12 & $4.14 \%$ \\
14 & $4.67 \%$ \\
\hline
\end{tabular}

\subsection{Comparison of Decryption Success between TCP and UDP}

The decryption result tends to decrease as the number of nodes increases. The decrease in the percentage of decryption in the TCP protocol tends not to be too large. However, in the UDP protocol, the percentage of successful decryption tends to be higher. In the results of sending data before encryption, the difference in the percentage of success between the TCP and UDP protocols is not too big. This can be seen in table 8 and figure 7 below:

Table 8. Comparison of the Success of Sent Data Packages Before the Encryption Process

\begin{tabular}{ccc}
\hline $\begin{array}{c}\text { Number of } \\
\text { Nodes }\end{array}$ & UDP Sent Data & TCP Sent Data \\
\hline 2 & $99 \%$ & $100 \%$ \\
4 & $98.50 \%$ & $100 \%$ \\
6 & $98.25 \%$ & $100 \%$ \\
8 & $97.80 \%$ & $99.40 \%$ \\
10 & $96.33 \%$ & $99.29 \%$ \\
12 & $95.86 \%$ & $99.14 \%$ \\
14 & $95.33 \%$ & $99.40 \%$ \\
16 & $97.30 \%$ & $99.20 \%$ \\
20 & $91.73 \%$ & $99.52 \%$ \\
\hline
\end{tabular}




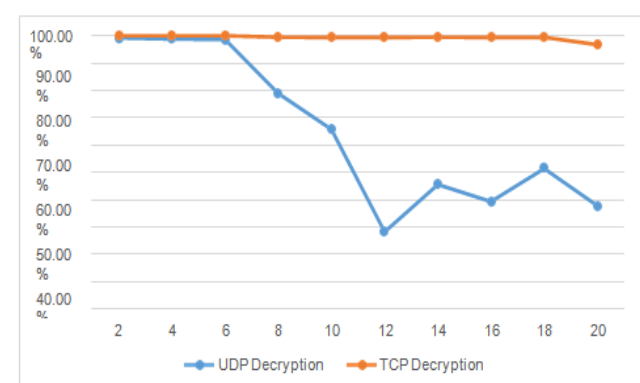

Figure 7. Comparison of Decryption Results with Arcfour Algorithm

Table 9.Comparison of Decryption Results with TripleDES Algorithm

\begin{tabular}{ccc}
\hline $\begin{array}{c}\text { Number of } \\
\text { Nodes }\end{array}$ & $\begin{array}{c}\text { UDP Decryption } \\
\text { Results }\end{array}$ & Decryption Result \\
\hline 2 & $99.36 \%$ & $100 \%$ \\
4 & $98.75 \%$ & $100 \%$ \\
6 & $98.45 \%$ & $100 \%$ \\
8 & $78.76 \%$ & $99.62 \%$ \\
10 & $65.63 \%$ & $99.54 \%$ \\
12 & $28.22 \%$ & $99.54 \%$ \\
14 & $45.46 \%$ & $99.64 \%$ \\
16 & $39.27 \%$ & $99.55 \%$ \\
18 & $53.30 \%$ & $99.61 \%$ \\
20 & $37.72 \%$ & $96.82 \%$ \\
\hline
\end{tabular}

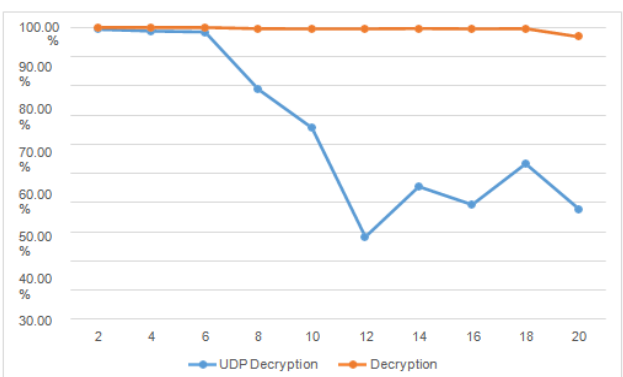

Figure 8. Comparison of Decryption Results with TripleDES Algorithm

\section{CONCLUSION}

The more nodes, the higher the packet loss. Both on TCP and UDP. Of the 3 algorithms used the difference in the results of the decryption is not much different.

The percentage of successful data transmission on TCP is greater with an average of $99.318 \%$ on the arcfour algorithm, $99.185 \%$ on the rijndael-192 algorithm, $99.132 \%$ on the tripledes algorithm. 
The percentage of successful data transmission on UDP is smaller with an average of $70.711 \%$ on the arcfour algorithm, $63.971 \%$ on the rijndael-192 algorithm, $64.412 \%$ on the tripledes algorithm.

The UDP protocol is not effective in sending data because of the low success rate of decryption.

\section{REFERENCES}

R. G. Hiertz, et. al., "The IEEE 802.11 universe," IEEE Communication Magazine, pp.62-70, Jan. 2010.

IEEE Computer Society, "Wireless LAN Medium Access Control (MAC) and Physical Layer (PHY) Specifications, Amendment 4: Enhancements for Very High Throughput for Operation in Bands below 6 GHz," IEEE Standard 802.11ac-2013: Part 11, Dec. 2013.

M. Arief, "Teknologi Jaringan Tanpa Kabel (Wireless)",Seminar Nasional Teknologi, 2007.

Zhou, Lidong, and Zygmunt J. Haas. "Securing ad hoc networks." IEE network 13.6 (1999).

Syafrizal, Melwin. Pengantar Jaringan Komputer. Penerbit Andi, 2005.

Adeputra Anugrah. 2009. Studi \& Implementasi Algoritma Triple DES.

Ferguson, P. \& Huston, G., 1998, Quality of Service, John Wiley \& Sons Inc.

Vassis, Dimitris, et al. "The IEEE $802.11 \mathrm{~g}$ standard for high data rate WLANs." Network, IEEE 19.3 (2005): 21-26 